\title{
Multicolour hologram based on plasmonic nanohole arrays and detour phase: design and simulation
}

\author{
Seyed Saleh Mousavi Khaleghi ${ }^{1}$, Dandan Wen ${ }^{1}$, Jasper Cadusch ${ }^{1}$, and Kenneth B. Crozier ${ }^{1,2,3}$ \\ 1. Department of Electrical and Electronic Engineering, University of Melbourne, Victoria 3010, Australia \\ 2. School of Physics, University of Melbourne, Victoria 3010, Australia \\ 3. ARC Centre of Excellence for Transformative Meta-Optical Systems (TMOS) \\ *Email: kcrozier@unimelb.edu.au
}

Multicolor holograms have numerous applications, including in art, data storage, security, and advanced displays. In recent years, there has been much interest on multicolor holograms based on metasurfaces [1]. Wen et al [2] demonstrated multicolour holograms using dielectric nanoparticles, with the nanoparticle size defining its resonant wavelength and its position defining its phase via the detour phase concept [3]. While this approach worked, it required good control over nanoparticle size and shape, achieved using inductively coupled reactive ion etching (ICP-RIE). This motivates the development of multicolour metasurface holograms that are also based on detour phase, but using other nanostructure types. Here we show that arrays of nanoholes in aluminium films enable the realization of multicolour holograms. We design nanohole arrays that serve as color (i.e. red/green/blue) filters with high transmission and low cross-talk. We then design two multicolour holograms based on these filters and simulate their performance, demonstrating that they show good fidelity to the desired holographic images. Our device is based on aluminum and silicon dioxide, giving it the advantage of CMOS compatibility.

Our hologram device consists of a glass substrate, on which aluminum (containing nanoholes) and $\mathrm{SiO}_{2}$ films are formed (Fig. 1a). Our hologram device is divided into pixels, each of which contains nanohole arrays (termed "nanohole groups") designed for red $(635 \mathrm{~nm})$, green $(532 \mathrm{~nm})$, and blue $(450 \mathrm{~nm})$ wavelengths. The nanohole diameters and spacings determine their transmission spectra. The position of the nanohole group in the $x$ direction determines the detour phase that the transmitted light carries. This is schematically illustrated for the red nanohole group in Fig. 1b, with the phase of the transmitted light being adjustable from $-\pi$ to $\pi$ by varying its position. In Fig. 1c (top panel), we show the simulated transmission spectrum for the pixel (i.e. structure of Fig. 1b). In Fig. 1c (bottom panel), we show the simulated transmission spectrum of nanohole arrays with the same periodicities and diameters as those of Fig. 1c (top panel), but for periodic arrays (rather than the pixel structure of Fig. 1b). It can be seen from Fig. 1c that high transmission efficiency and low cross talk are achieved.

We next design two Fourier-type detour phase holograms using our nanohole pixels. First, we divide the target image (top panels of Fig. 1d) into three sub-images that correspond to red, green, and blue (RGB). Second, we use the Gerchberg-Saxton algorithm to design three holograms (for RGB) with equal sizes. The three holograms are combined into one hologram, where the phases needed for the RGB channels are encoded by the positions of the nanohole groups. It can be seen that the holographic images predicted to be produced by our nanohole devices (bottom panels of Fig. 1d) are in good agreement with the intended designs (top panels of Fig. 1d). Some speckle is predicted, but could be removed by filtering the high frequencies and other techniques mentioned in [4].
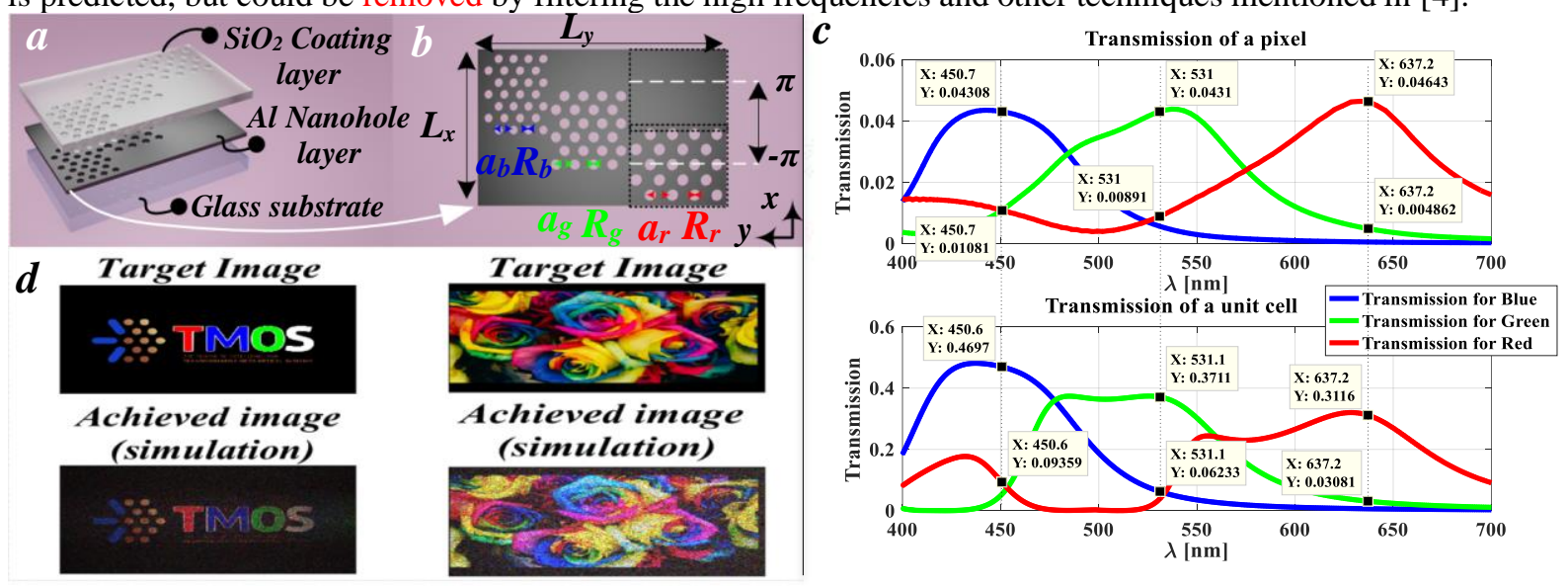

Fig. 1 (a) Schematic of hologram pixel. $\mathrm{Al}$ and $\mathrm{SiO}_{2}$ layers are $150 \mathrm{~nm}$ and $200 \mathrm{~nm}$ thick, respectively. (b) Schematic of single pixel, showing blue, green and red nanohole groups. Geometric parameters: $a_{r}=395 \mathrm{~nm}, R_{r}=210 \mathrm{~nm}, a_{g}=320 \mathrm{~nm}, R_{g}=175 \mathrm{~nm}, a_{b}=$ $255 \mathrm{~nm}, R_{b}=145 \mathrm{~nm}, L_{x}=3000 \mathrm{~nm}, L_{y}=4350 \mathrm{~nm}$. (c) Transmission spectra of pixel (top panel) and of periodic arrays (bottom panel).. (d) Top panels: target images. Bottom panels: predicted holographic images.

\section{References}

[1] Wen, D., Cadusch, J. J., Meng, J., \& Crozier, K. B. (2021). Light field on a chip: metasurface-based multicolor holograms. Adv Photonics (submitted)

[2] Wen, D., Cadusch, J. J., Meng, J., \& Crozier, K. B. (2020). Multifunctional dielectric metasurfaces consisting of color holograms encoded into color printed images. Adv Functional Materials, 30(3), 1906415.

[3] J. W. Goodman, Introduction to Fourier Optics, Roberts and Company Publishers, Greenwood Village, CO 2005

[4] Bianco, V., Memmolo, P., Leo, M., Montresor, S., Distante, C., Paturzo, M., \& Ferraro, P. (2018). Strategies for reducing speckle noise in digital holography. Light: Science \& Applications, 7(1), 1-16. 


\section{University Library}

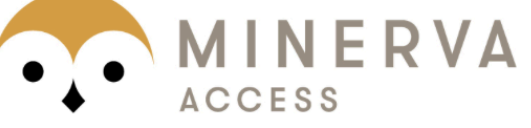

A gateway to Melbourne's research publications

Minerva Access is the Institutional Repository of The University of Melbourne

Author/s:

Khaleghi, SSM;Wen, D;Cadusch, J;Crozier, KB

Title:

Multicolor hologram based on plasmonic nanohole arrays and detour phase: design and simulation

Date:

2021-01-01

\section{Citation:}

Khaleghi, S. S. M., Wen, D., Cadusch, J. \& Crozier, K. B. (2021). Multicolor hologram based on plasmonic nanohole arrays and detour phase: design and simulation. [Abstract]. 2021 CONFERENCE ON LASERS AND ELECTRO-OPTICS EUROPE \& EUROPEAN QUANTUM ELECTRONICS CONFERENCE (CLEO/EUROPE-EQEC), 00, https://doi.org/10.1109/CLEO/ Europe-EQEC52157.2021.9541675.

Persistent Link:

http://hdl.handle.net/11343/294846 